\title{
Challenges in Acanthamoeba keratitis - treatment efficacy of a sight-threatening disease in a severe case without identified risk factors requiring diagnostic verification
}

\author{
Agnieszka Kuligowska ${ }^{1, A-D, F \oplus}$, Wanda Baltaza ${ }^{2, B, D, F} \oplus$, Lidia Chomicz ${ }^{2,3, C-F \oplus}$, Anna Machalińska ${ }^{1, A, C, E-F \oplus ~}$ \\ ${ }^{1}$ First Department of Ophthalmology, Pomeranian Medical University, Szczecin, Poland \\ 2 Department of Medical Biology, Medical University of Warsaw, Warsaw, Poland \\ ${ }^{3}$ SPKSO Ophthalmic Hospital, Warsaw, Poland \\ A - Research concept and design, B - Collection and/or assembly of data, C - Data analysis and interpretation, \\ $D$ - Writing the article, $E$ - Critical revision of the article, F - Final approval of the article
}

\begin{abstract}
Kuligowska A, Baltaza W, Chomicz L, Machalińska A. Challenges in Acanthamoeba keratitis - treatment efficacy of a sight-threatening disease in a severe case without identified risk factors requiring diagnostic verification. Ann Agric Environ Med. 2021; 28(4): 719-723. doi: 10.26444/ aaem/138635
\end{abstract}

\begin{abstract}
Acanthamoeba keratitis (AK), the vision-threatening disease caused by the amphizoic, potentially parasitic amoebae is growing threat for public health in Poland and worldwide. The report presents the case of 70-year-old man with severe keratitis admitted to an Ophthalmology Clinic. Before admission, the patient had been treated for 6 months with antibacterial and antifungal drugs in other units, without improvement in the eye condition. The use of in vivo confocal microscopy and in vitro cultivation allowed diagnosis to be verified and AK successfully treated. Awareness of the threat to public health caused by Acanthamoeba spp is still insufficient. If there is failure in response to first line therapy, AK should be taken into account, despite the lack of identified risk factors. In vitro monitoring of amoebic strain can be helpful for prognosis of the course of the corneal disease. Improvement in duration from first symptoms until proper diagnosis is decisive for better treatment efficacy.
\end{abstract}

\section{- Key words}

Acanthamoeba keratitis, diagnostic techniques and procedures, confocal microsycopy, light microscopy, axenic culture, combination drug therapy

\section{INTRODUCTION}

The free-living protists of Acanthamoeba spp., known as potential agents of disease in humans, are widespread in various outdoor environments in many parts of the world $[1,2]$. Devastating eye disease caused by amoebic infection detected nowadays with increasing frequency is an emerging threat to public health in Poland and worldwide [3-8].

The amoebae exist in two morphological forms: an active vegetative stage - the trophozoite, and in a dormant stage the cyst, indicating minimal metabolic activity. The amoebae complete their life cycles in natural environments: in air, soil, dust, and different aquatic habitats; they are also found in/on vegetables and various animal organisms. The free -living amoebae (FLA) have been also isolated from manmade environments; they are able to survive in chlorinated swimming pools and air conditioning units, as well as in domestic tap water and municipal sewage systems; they have also been detected on surfaces of different equipment and accessories in a hospital environment, [2, 4, 9-13].

Some amoeboid protists of Acanthamoeba genus are considered amphizoic because under predisposing

Address for correspondence: Lidia Chomicz, Department of Medical Biology, Medical University of Warsaw, Litewska 14/16, 00-575 Warsaw, Poland. E-mail: lidia.chomicz@wum.edu.pl;

Anna Machalińska, First Department of Ophthalmology, Pomeranian Medical University, Al. Powstancow Wlkp. 72, 70-111 Szczecin, Poland.

E-mail: annam@pum.edu.pl

Received: 01.05.2021; accepted: 08.06.2021; first published: 05.07.2021 circumstances they are able to enter the human body from different sources and exist as parasites causing pathogenic effects on various human organs and tissues.

In particular, potentially parasitic Acanthamoeba strains can cause the severe, vision-threatening corneal disease. Acanthamoeba keratitis (AK) [1-4, 6-10].

Risk factors. Wearing contact lenses is considered a leading risk factor for AK, especially poor contact lens hygiene, use of contact lenses during bathing, cleaning them with tap water; micro-injuries to the cornea also promote the eye infection $[4,9-13]$. The AK can also occur in persons not using contact lenses. Nowadays, such cases of the vision-threatening disease occur more frequently [12-14]. The corneal epithelial cells, trauma and ocular surgery are also risk factors of AK, especially important in non-contact lens users. In addition, exposure of the eye to water containing amphizoic amoebae, e.g. in natural water bodies and swimming pools are also circumstances predisposing to Acanthamoeba infections in humans [9-11].

Clinical picture. The earliest signs of AK include non-specific symptoms: redness, photophobia, excessivelacrymation and progressive visual impairment. Although only one eye is usually affected, bilateral infections have also been reported. In the vision-threatening disease, there may be diffuse irregular epithelial oedema which can eventually progress to a dendriform ulcer. If $\mathrm{AK}$ is left untreated, the amoebae 
invade all layers of the cornea, forming a ring abscess, and inducing scarring and corneal ulceration which may lead to corneal perforation [10-16]. Without suitable treatment, this severe, prolonged, sight-threatening human disease may lead to corneal necrosis and blindness.

Non-specific clinical symptoms similar to these observed in viral, fungal or bacterial keratitis, are a common cause of misdiagnoses and ineffective therapy of AK. Certain clinical features prompt the identification of Acanthamoeba infection, i.e. anterior paracentral stromal infiltration, as a partial or complete circle with a clear central cornea; patients also often complain of unbearable pain [9-13, 15]. If a presence of a foreign particle has been excluded, the occurrence of these symptoms may suggest AK due to radial keratoneuritis associated with Acanthamoeba trophozoite activeness; however, pain does not always occur.

Non-invasive in vivo methods, the slit-lamp and confocal microscopy, are used to diagnose Acanthamoeba keratitis. Corneal erosions, ulceration and the presence of ring-like corneal infiltration (in less than $20 \%$ of patients) may be visualized by the slit lamp in the affected eye.

Confocal microscopy, apart from being non-invasive, is a relatively simple, highly sensitive method for rapid in vivo $\mathrm{AK}$ diagnosis [13, 15-18]. Hyper-reflective objects, presumably cysts and trophozoites of Acanthamoeba, can be revealed by this technique. However, it should be taken into account that no amoebae will be visible if a low density of protists occurs in the infected eye, or if there are mixed infections; it should therefore be applied for a tentative diagnosis. Because of the wide range of non-specific symptoms, it is emphasized that diagnosis based on clinical symptoms alone is not sufficient to determine the causative agent of the infectious keratitis in humans $[9,10,13]$.

Direct detection of the causative agents - a visualizing of amoeba developmental forms, is very important for the proper diagnosis as well as for verification of common misdiagnose. Laboratory examinations of material from corneal scrapings in contrast-phase light microscopy allow visualization /identifation of Acanthamoeba cysts and/or trophozoites, and to verification of previous misdiagnoses [7, $9,13]$. Also, in vitro cultivated samples acquired from corneal scrapings to obtain more material for further research, e.g. for examination in terms of diagnostics verification and monitoring of the morpho-physiology of the Acanthamoeba population $[13,18,19]$.

Identification of the corneal strains to species level can also be performed by PCR, and molecular techniques based on genotype associations of the $18 \mathrm{~S}$ rRNA gene sequences, $[9,10,13]$; however, the technology is not readily available everywhere.

The treatment of AK has not yet been fully established. Combined drug therapy mainly involves the topical application of chlorhexidine digluconate, propamidine isethionate (Brolene) and polyhexamethylene biguanide (PHMB), with an addition of antibiotics to avoid possible bacterial infections. Effects of the applied therapy are often disappointing due to misdiagnoses and the extremely high resistance of Acanthamoeba cysts to chemicals, antimicrobial and anti-parasitic drugs [9-11, 13-19].

During the last few decades, Acanthamoeba keratitis, the vision-threatening corneal disease is being reported in Poland with constantly increasing frequency $[7,10,18]$.

This retrospective report presents the case of patient with serious visual acuity impairment in the course of improperly treated severe keratitis. The study was performed in accordance with the tenets of the Declaration of Helsinki. There were no identified risk factors, and the beginning of an appropriate treatment was delayed due to the earlier misdiagnosis.

The case is evaluated in terms of clinical, diagnostic and treatment aspects.

\section{CASE REPORT}

A 70-year-old man was admitted to the Ophthalmology Clinic after undergoing unsuccessful treatment of severe keratitis for six months in other ophthalmic units. The patient complained of intermittent pain, blurred vision, lacrymation, strong photophobia, and significant congestion of the left eyeball. His medical history regarding eye diseases was negative, the patient only reported symptoms of intermittent mild dry eye disease. The patient did not wear contact lenses, had no history of eye injuries, and also denied swimming in any water reservoirs or working with soil.

Before admission to the clinic, during the six-month diagnostic and therapeutic process, the patient was treated with numerous preservative-free moisturizing eye drops (he did not remember the name), almost all available ophthalmic topical antibiotics (consecutively or in combination therapy), among others: ofloxacin four times daily, moxifloxacin four to six times daily, levofloxacin and tobramycin six times daily (administered simultaneously), as well as fortified gentamicin $1.5 \%$ and vancomycin 5\% every hour with a break at night. $\mathrm{He}$ also received oral acyclovir $400 \mathrm{mg}$ and topical ganciclovir five times daily anti-viral treatment. Anti-fungal eyedrops were also applied - amphotericin B and voriconazole unfortunately the dosing frequency is unknown. The patient also reported receiving subconjunctival injections which consisted of biodacin and dexamethasone mixed in a ratio of 1:1 several times just before admission to the clinic. He additionally mentioned short-term topical dexamethasone therapy, but the frequency is unknown.

On the admission to the First Department of Ophthalmology at the Pomeranian Medical University in Szczecin,northern Poland, the patient's visual acuity was limited to the perception of light in the left eye and 1.0 on the Snellen chart in the right eye. He had a severe course of keratitis. After the previous ineffective anti-microbial treatment, urgent diagnostic verification was necessary during which Acanthamoeba spp infection as an etiological factor of the corneal disease was taken into account.

Examination of the anterior segment revealed extensive ring-shaped infiltration involving two-thirds of the corneal surface, accompanied by severe ciliary congestion and corneal neovascularization due to limbal barrier breakdown. (Fig. 1A). Anterior segment optical coherent tomography (AS-OCT) revealed thickening of the cornea, hypopyon in the anterior chamber, and inflammatory cells on the endothelium. Non-invasive in vivo confocal microscopy revealed multiple Acanthamoeba cysts in the corneal epithelial layer (Fig. 1B). Stimulation of the epithelial cell nuclei was also observed.

During laboratory parasitological examinations, scrapings from the corneal epithelium of the affected eye were collected and parasitologically examined to confirm 


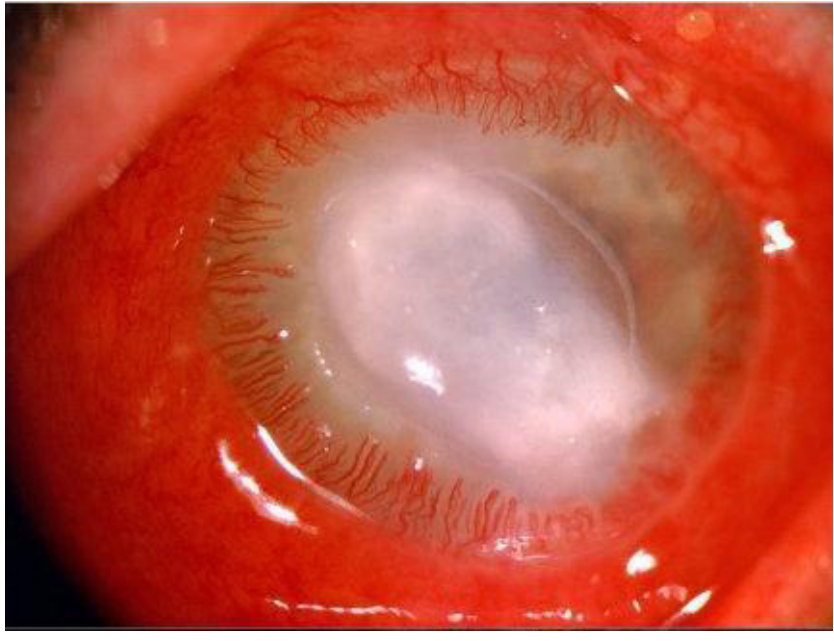

Figure 1A. Ring-shaped extensive infiltration covering $2 / 3$ of the corneal surface, severe ciliary congestion and corneal neovascularization caused by infection with Acanthamoeba sp. - slit-lamp photograph

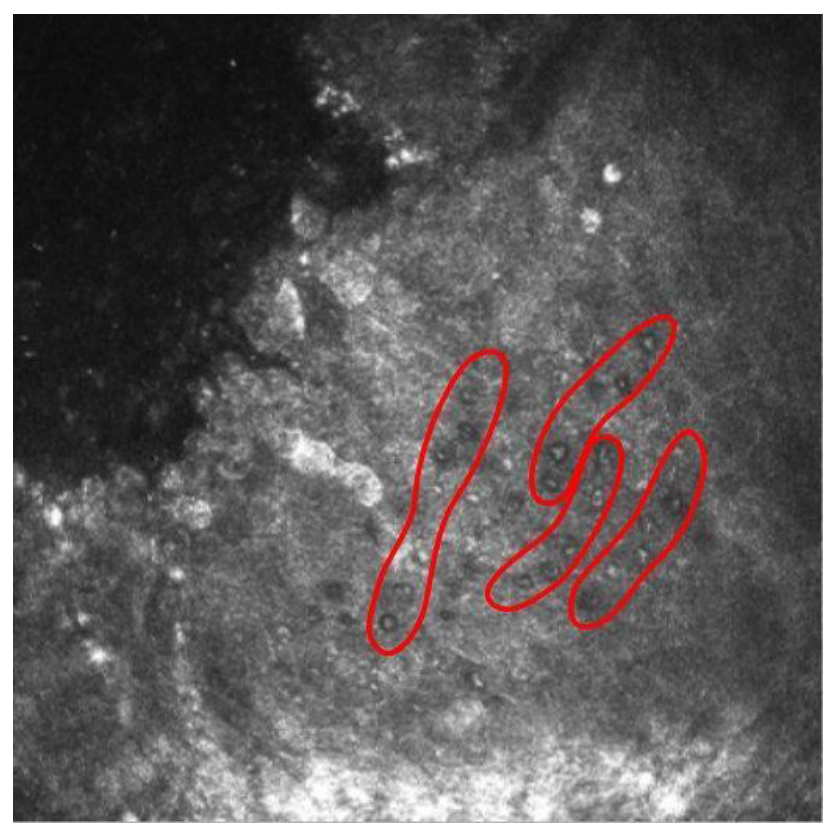

Figure 1B. Red circles indicate the presence of hyper-reflective objects Acanthamoeba cysts arranged in characteristic 'chains' in the corneal epithelium of the affected eye - in vivo confocal microscopy
Acanthamoeba keratitis. Identification of the protist was based on its morpho-physiology. In the contrast- phase light microscope (100 $\times$ and $400 \times$ magnifications) double-walled, rounded Acanthamoeba cysts, $10-20 \mu \mathrm{m}$ in size and live, changing in shape trophozoites, $18-40 \mu \mathrm{m}$ in size, moving by acanthopodia with spine-like protrusions were detected.

Samples of the Acanthamoeba corneal isolates were also in vitro grown under bacteria-free condition. Cultivation of this corneal amoebic strain was performed as in earlier research in BSC medium composed of Bacto Casitone, Difco, with $10 \%$ calf serum (Wytwórnia Surowic i Szczepionek, Lublin, Poland) with the addition of an aqueous solution of antibiotics, at $26^{\circ} \mathrm{C}$, and regularly sub-cultured twice a month [18-20]. The in vitro cultivation was performed in the laboratory of the Department of Medical Biology at the Medical University in Warsaw. The culture was maintained as long as the isolate survived. Samples of the culture were examined with a light microscope to visualize and identify amoebae, based on their morphology.

The in vitro monitoring of cultivated amoebic strain was a useful tool for evaluating its viability and changes in morphophysiological dynamics. The viability of Acanthamoeba trophozoites was expressed as the ability to multiply. Monitoring of the amoeba population revealed gradually weakening in vitro developmental dynamics of this corneal strain; after five cycles of sub-culturing (2.5 months), no dividing trophozoites were found.

Treatment was initiated consisting of the administration of $0.02 \%$ chlorhexidine and $0.1 \%$ propamidine hourly during the daytime and every three hours at night. After five days of treatment, the hypopyon had disappered, corneal melting ceased, and the degree of infiltration decreased. The patient also reported significant improvement in his eye condition. The initial dose was used for two additional days and then reduced to one drop every two hours during the day and every four hours at night. From the third week, topical treatment was used four times daily and twice at night.

After six weeks of treatment, a significant reduction of corneal oedema, decrease in the magnitude of infiltration, and improvement in the corneal structure were reported (Fig. 2A). The patient's visual acuity improved to counting fingers. Since the patient reported burning and itching after using chlorhexidine drops, administration was stopped. Because aggressive corneal neovascularization did not

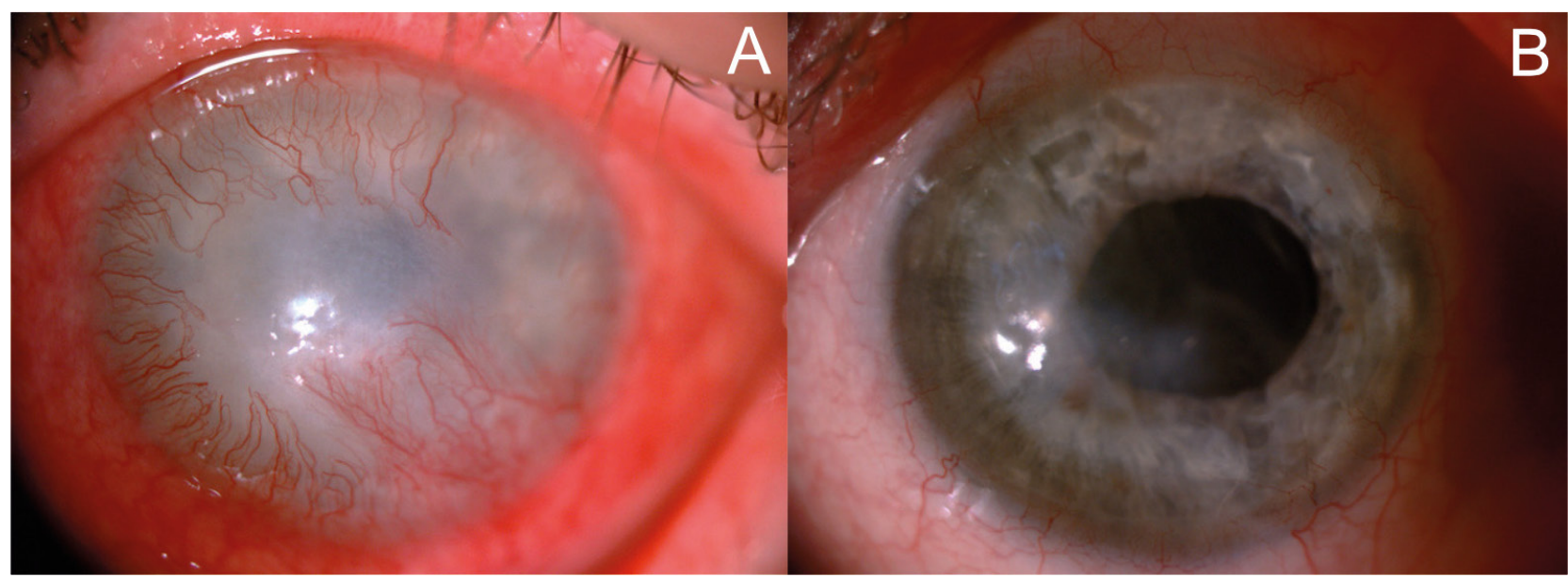

Figure 2. Clinical examination after 6 weeks of treatment (A) and at the end of treatment (B) - slit-lamp images. 
respond to conventional therapy, bevacizumab eye drops at a $5 \mathrm{mg} / \mathrm{ml}$ concentration five times a day were prescribed for 14 days [21-23]. Propamidine was continued. A slight reduction in limbal congestion was noticed. Vessel ingrowth was inhibited. In the eighth week of treatment, due to corneal haze, dexamethasone eye drops were administered, and the dosage was increased weekly (starting from $1 x$ daily to $4 \mathrm{x}$ daily over four weeks). The patient was examined each week before the dosage was increased. Improvements were noted, and visual acuity stabilized at 0.01 . The corneal surface was also stable. The propamidine dose was reduced to $2 \mathrm{x}$ daily, while the steroid dosage was maintained at $4 \mathrm{x}$ daily.

After six months of the therapy, visual acuity gradually improved to 0.3 on the Snellen chart, and the corneal condition and translucency significantly improved. AS-OCT scans revealed a stable corneal surface and the presence of a hyperreflective scar covered with healthy epithelium. Due to the presence of post-inflammatory cataract and posterior circular synechiae, cataract surgery was performed. No major complications were observed.

At a check-up visit, roughly one month after the operation, there were no signs of inflammation, the vessels had regressed, and the corneal surface completely covered with healthy epithelium. There was a scar located in the anterior corneal stroma; the cornea was translucent (Fig. 2B); the anterior chamber was clean, and there were no pathological vessels in the iris; the pupil had a medium width and reacted lazily to light, and the artificial lens was in the correct position. The best corrected visual acuity was 0.6 on the Snellen chart. The patient's quality of life improved significantly. The antiAcanthamoeba treatment was stopped and intensity of the steroid treatment was gradually reduced. DALK surgery was suggested to the patient, but due to the satisfying visual acuity, he declined. Currently, the only medicine that patient uses are artificial tears without preservatives.

\section{DISCUSSION}

The threat generated by amphizoic, facultative parasitic strains of Acanthamoeba genus is an emerging medical problem worldwide. The number of cases of this disease of the human eye that can result in ulceration of the cornea, loss of visual acuity, and even blindness, is growing each year in many parts of the world [3-6, 11-16]. In Poland, Acanthamoeba keratitis is still considered a rare disease, difficult to diagnose and treat, and generates a serious threat to public health $[7,10,18,19]$. Potentially pathogenic Acanthamoeba species are ubiquitous and widely distributed in natural and man-made environments; thus, prevention is very difficult as humans are frequently exposed to the amoebae. AK is difficult to diagnose because their clinical symptoms are non-specific and similar to those observed in the course of other infectious eye diseases.

It is a fact that the most common factors predisposing to contract $\mathrm{AK}$ are related to contact lens wear. Although more than $85 \%$ of all incidences have been recognized in different countries in wearers of contact lenses, this corneal disease is also detected in persons not using lenses $[9,10,14]$.

The presented case has analyzed the case of patient who did not wear contact lenses who presented serious challenges for clinicians due to severe keratitis. During the six-month diagnostic and therapeutic process before admission to the clinic, the patient had been treated in other ophthalmic units with antibacterial and antifungal drugs, with no improvement in the eye condition.

This case presented a challenge in term of diagnosis and treatment because of:

1) lack of identified risk factors in medical history;

2) wide range of non-specific symptoms;

3) initially incorrect diagnosis;

4) significant delay in correct diagnosis;

5) delay in beginning appropriate treatment.

The Juárez et al. [16] emphasized that 'there are two principal causes that lead to severe outcomes: misdiagnosis or late diagnosis of the causal agent, and lack of a fully effective therapy due to the existence of a highly resistant cyst stage of Acanthamoeba.'

Diagnostic errors that delayed appropriate treatment affected the prolonged and severe course of the keratitis; therefore, a quick and accurate verified diagnosis was necessary to ensure effective treatment and a good prognosis. An important aspect of the proper diagnosis and treatment of $\mathrm{AK}$ is a carefully collected medical history, including the identified risk factors. In persons not using contact lenses there are other important risk factors for acquiring AK that can include a corneal epithelial injury, eye surgery, exposure of the eye to water or moist soil in which Acanthamoeba forms exist $[11,13,15,20]$.

In the presented case, previously unsuccessfully treated, urgent diagnostic verification was performed; Acanthamoeba spp. as the etiological agent of a serious corneal disease was been taken into account despite the lack of identified risk factors. Earlier, the patient reported dry eye episodes. In this case, it was only suspected that Acanthamoeba infection was the most likely the consequence of intense eye scratching, as well as exposure to contaminated tap water when washing and cleaning the face.

Presumptive diagnosis can be made through clinical features and in vivo confocal microscopy. This was used in the presented case for the diagnostic verification procedure: numerous hyper-reflective objects, presumably Acanthamoeba cysts and trophozoites, were revealed by this in vivo technique.

Literature data indicate that in vivo confocal microscopy is currently the preferred non-invasive technique for specific diagnosis by visualizing the infectious pathogens within the corneal tissues, e.g. Acanthamoeba cysts and trophozoites. The full thickness of the cornea can be scanned by in vivo confocal microscopy with no risk for the patient. It allows evaluation of the progress of the therapeutic process and determine its completion, and when treatment is no longer necessary $[6$, $9,13,16-20]$. This valuable tool for a presumptive specific diagnosis of eye disease shows approximately $90 \%$ sensitivity; however, its use demands an experienced examiner in order to avoid false results. Moreover, the limited availability of confocal microscopy represents a substantial limitation of this non-invasive technique.

The definitive diagnosis can be made on the basis of in vitro cultivation techniques which, however, need close collaboration between clinicians and laboratory staff. The method is considered as the gold standard of accurate diagnosis that allows for a direct visualizing of Acanthamoeba developmental forms $[13,16,21]$. In the presented case, the in 
vitro monitoring was useful for confirmation of the proper diagnosis of AK; simultaneously, a low protist number and poor in vitro viability of the corneal strain population was observed after appropriate treatment hds been applied.

Current therapies against AK have not been fully established. Mainly, they involve topical application of chlorhexidine digluconate, propamidine isethionate and polyhexamethylene biguanide, with an addition of antibiotics because bacterial co-infections are possible. Results of applied therapy are often disappointing due to the resistance of amoebae to disinfectants and drugs. It is emphasized that the extremely high resistance of double-walled Acanthamoeba cysts to chemicals, anti-microbial and anti-pearasitic drugs, is considered one of the key contributors to treatment failure and prolonged course of the disease. It is worth mentioning that the use of topical steroids prior to applying intensive anti-amoebic therapy is associated with a worse prognosis, and may also delay a proper diagnosis [24]. Moreover, corticosteroids exacerbate Acanthamoeba infection and promote recrudescence by inducing the excystment of dormant cysts and stimulating proliferation and the activation of emerging trophozoites.

In the opinion of the authors of this case report, steroids are useful in alleviating the inflammation and pain that accompany Acanthamoeba keratitis, but they should be administered only after positive effects associated with the use of anti-parasitic drugs are observed.

\section{CONCLUSIONS}

The medical history presented in this case report proves that knowledge and awareness of the increasing public health threat generated by amphizoic amoebae as the causative agents of a vision-threatening disease, Acanthamoeba keratitis (AK), is still insufficient. AK should be taken into account despite the lack of identified risk factors when there is a failure in response to first line therapy for anti-bacterial or anti-fungal agents. An improvement in duration from first symptoms until proper diagnosis of $\mathrm{AK}$ is decisive for better treatment procedure effectiveness. The in vitro cultivation methods and monitoring of amoebic strain viability can be helpful for prognosis of the corneal disease course and the treatment efficacy; however, good collaboration between clinicians and laboratory staff is necessary. In severe cases, keratoplasty and prolonged application of a mixture of drugs may be an appropriate option for visual rehabilitation. Further research and educational efforts are desirable for the prevention of the threat from this vision-threatening corneal disease.

\section{REFERENCES}

1. Marciano-Cabral F, Cabral G. Acanthamoeba spp. as agents of disease in humans. Clin Microbiol Rev. 2003; 16(2): 273-307. https://doi. org/10.1128/cmr.16.2.273-307.2003

2. Schuster FL, Visvesvara GS. Free-living amoebae as opportunistic and non-opportunistic pathogens of humans and animals. Int J Parasitol. 2004; 34: 1001-1027.
3. McKelvie J, Alshiakhi M, Ziaei M, et al. The rising tide of Acanthamoeba keratitis in Auckland, New Zealand: a7-year review of presentation, diagnosis and outcomes (2009-2016). Clin Exp Ophthalmol. 2018; 46: 600-607. https://doi.org/10.1111/ceo.13166

4. Hajialilo E, Niyyati M, Solaymani M, et al. Pathogenic free-living amoebae isolated from contact lenses of keratitis patients. Iran J Parasitol. 2015; 10(4): 541-546.

5. Walochnik J, Scheikl U, Haller-Schober EM. Twenty years of Acanthamoeba diagnostics in Austria. J Eukaryot Microbiol. 2015; 62: 3-11. https://doi.org/10.1111/jeu.12149

6. Huang,FC, Shih MH, Chang KF, et al. Characterizing clinical isolates of Acanthamoeba castellanii with high resistance to polyhexamethylene biguanide in Taiwan. J Microbiol Immunol Infect. 2017; 50: 570-577. doi: S1684-1182(15)00908-1 [pii].1155/2015/231285

7. Chomicz L, Conn DB, Padzik M, et al. Emerging threats for human health in Poland: pathogenic isolates from drug resistant Acanthamoeba keratitis monitored in terms of their in vitro dynamics and temperature adaptability. BioMed Res Int. 2015, ID231285. https://doi.org/10.

8. Król-Turmińska K, Olender A. Human infections caused by freeliving amoebae. Ann Agric Environ Med. 2017; 24(2): 254-260. doi: $10.5604 / 12321966.1233568$

9. Khan NA. Acanthamoeba: biology and pathogenesis. 2nd ed. Caister Academic Press; 2015.

10. Chomicz L, Szaflik JP, Padzik M, Izdebska J. Acanthamoeba keratitis: the emerging vision threatening corneal disease. In: Advances in Common Eye Infections. (Ed. S. Rumelt). INTECH. 2016, Chapter 4, p. 99-120.

11. Somani SN, Ronquillo Y, Moshirfar M. Acanthamoeba Keratitis. In Stat. Pearls. Publishing LLC: Treasure Island, FL, USA, 2020.

12. Shimmura-Tomita M, Takano H, Kinoshita N, et al. Risk factors and clinical signs of severe Acanthamoeba keratitis. Clin Ophthalmol. 2018; 12: 2567-2573.

13. Lorenzo-Morales J, Khan NA, WalochnikJ. An update on Acanthamoeba keratitis: diagnosis, pathogenesis and treatment. Parasite. 2015; 22(10): 1-20. https://doi.org/10.1051/parasite/2015010

14. Garg P, Kalra P, Joseph J. Non-contact lens related Acanthamoeba keratitis. Indian J Ophthalmol. 2017; 65(11): 1079-1086. https://oi. org/10.4103/ijo.IJO_826_17

15. Szentmary N, Daas L, Shi L, et al. Acanthamoeba keratitis - Clinical signs, differential diagnosis and treatment. J Current Ophthalmol. 2019; 31: 16-23.

16. Juárez MM, Tártara LI, Cid AG, et al. Acanthamoeba in the eye, can the parasite hide even more? Latest developments on the disease. Contact Lens \& Anterior Eye. 2018; 41: 245-251. doi: 10.1016/j.clae.2017.12.017

17. Villani E, Baudouin C, Efron N, et al. In vivo confocal microscopy of the ocular surface: from bench to bedside. Curr. Eye. Res. 2014; 39: 213-231. doi: 10.3109/02713683.2013.842592

18. Padzik M, Szaflik JP, Baltaza W, et al. In vivo confocal microscopy and in vitro culture techniques as tools for evaluation of severe Acanthamoeba keratitis incidents. Ann Parasitol. 2017; 63(4): 341-346.

19. Chomicz L, Padzik M, Szaflik JP, et al. Monitoring of in vitro dynamics of Acanthamoeba strains isolated from infected eyes as an useful tool in keratitis management. Exp Parasitol. 2014; 145: 73-77.

20. Červa L, Novak K. Amoebic meningoencephalitis: sixteen fatalities. Science. 1968; 160: 92.

21. Sepehr Feizi. Corneal Angiogenesis: Etiologies, Complications, and Management, In: Physiologic and Pathologic Angiogenesis - Signaling Mechanisms and Targeted Therapy, (Ed. Dan Simionescu and Agneta Simionescu), IntechOpen. 2017. Available at: https://www.intechopen. com/books/physiologic-and-pathologic-angiogenesis-signalingmechanisms-and-targeted-therapy/corneal-angiogenesis-etiologiescomplications-and-management

22. Bock F, Konig Y, Kruse F, et al. Bevacizumab (Avastin) eye drops inhibit corneal neovascularization. Graefe's Arch Clin Exp Ophthalmol. 2008; 246(2): 281-284. https://doi.org/10.1007/s00417-007-0684-4

23. Koenig Y, Bock F, Horn F, et al. Short- and long-term safety profile and efficacy of topical bevacizumab (Avastin) eye drops against corneal neovascularization. Graefe's Arch Clin Exp Ophthalmol. 2009; 247(10): 1375-1382. https://doi.org/10.1007/s00417-009-1099-1

24. Robaei D, Carnt N, Minassian DC, et al. The impact of topical corticosteroid use before diagnosis on the outcome of Acanthamoeba keratitis. Ophthalmol. 2014; 121(7): 1383-1388. https://doi.org/10.1016/j. ophtha.2014.01.031 